HETEROCYCLES, Vol. 99, No. 2, 2019, pp. 989 - 1002. ( 2019 The Japan Institute of Heterocyclic Chemistry

Received, 8th September, 2018, Accepted, 3rd October, 2018, Published online, 22nd November, 2018

DOI: $10.3987 / C O M-18-S(F) 63$

\title{
ENANTIOSELECTIVE SYNTHESIS OF 2,4,5-TRISUBSTITUTED TETRAHYDROPYRANS VIA PEPTIDE-CATALYZED MICHAEL ADDITION FOLLOWED BY KISHI'S REDUCTIVE CYCLIZATION
}

\author{
Atsushi Ueda,* Mei Higuchi, Tomohiro Umeno, and Masakazu Tanaka* \\ Graduate School of Biomedical Sciences, Nagasaki University, 1-14 \\ Bunkyo-machi, Nagasaki 852-8521, Japan. E-mail: aueda@nagasaki-u.ac.jp (A. \\ Ueda).
}

\begin{abstract}
An enantioselective synthesis of 2,4,5-trisubstituted tetrahydropyrans has been achieved in four steps from $\alpha, \beta$-unsaturated ketones and dimethyl malonate by peptide-catalyzed asymmetric Michael addition and diastereoselective construction of tetrahydropyran rings by Kishi's reductive cyclization as key steps. A variety of $\alpha, \beta$-unsaturated ketones were converted to the 1,4-products with high enantioselectivities (83-98\% ee).
\end{abstract}

\section{INTRODUCTION}

A number of isolations as well as syntheses of biologically active natural products possessing tetrahydropyran (THP) rings were reported, for example, laulimalides, ${ }^{1}$ bryostatins, ${ }^{2}$ halichondrins, ${ }^{3}$ and so on. Therefore, there are many efforts at developing enantioselective synthesis of THP ring motifs. ${ }^{4} \mathrm{We}$ recently reported an enantioselective synthesis of 2,4,5-trisubstituted THP 5, which involves: (1) helical peptide 1-catalyzed enantioselective Michael addition reaction of dimethyl malonate to enone 2 with $94 \%$ ee; (2) ketal protection of $\mathbf{3}$ with neopentyl glycol and subsequent reduction of ester moiety to give diol $\mathbf{4}$; (3) Kishi's reductive cyclization ${ }^{5}$ using unprecedented prochiral 1,3-diol as a precursor to afford the desired THP 5 as a single diastereomer without loss of the enantiomeric purity (Scheme 1). ${ }^{6}$ It is important to note that two hydroxy groups in prochiral diol $\mathbf{4}$ were completely differentiated to create new stereogenic centers at $\mathrm{C} 2$ and $\mathrm{C} 5$ positions. This high diastereoselectivity could be derived from phenyl substituent at $\mathrm{C} 4$ position. ${ }^{7}$ Since peptides are promising organocatalyst to construct new stereogenic center with a broad scope of substrates, ${ }^{8}$ this four-step protocol to construct 2,4,5-trisubstituted THP motif could be applicable to various substrates. Herein, we report an enantioselective synthesis of 2,4,5-trisubstituted THPs.

This paper is dedicated to Professor Tohru Fukuyama on the occasion of his 70th birthday. 

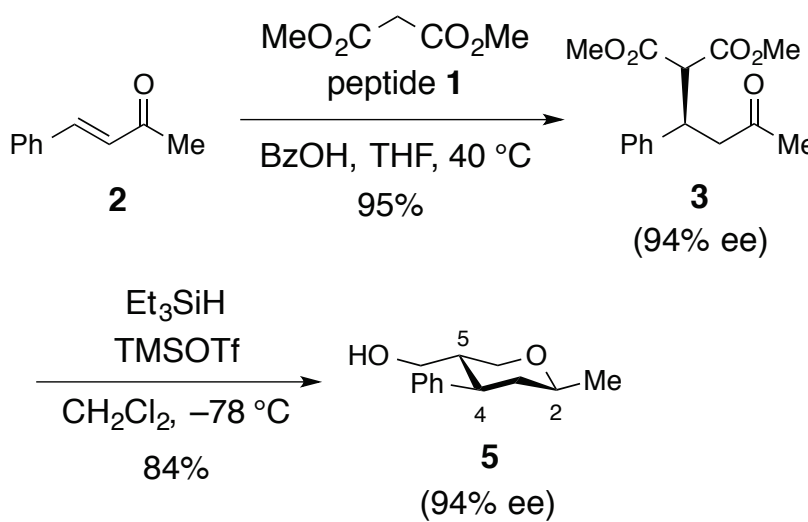


Scheme 1. Stereoselective construction of 2,4,5-trisubstituted THP 5

\section{RESULTS AND DISCUSSION}

We first investigated the influence of substitution patterns on the $\mathrm{R}^{1}$ phenyl ring for the peptide-catalyzed Michael reaction. ${ }^{9}$ Both $\mathbf{6 a}$ possessing an electron-withdrawing group and $\mathbf{6 b}$ possessing an electron-donating group provided excellent enantioselectivities and yields (98\% ee and 95\% ee, Table 1, entries 1 and 2). 2-Furyl and 2-naphthyl derivatives were also suitable substrates for these peptide

Table 1. Substrate scope for the peptide-catalyzed Michael addition reaction

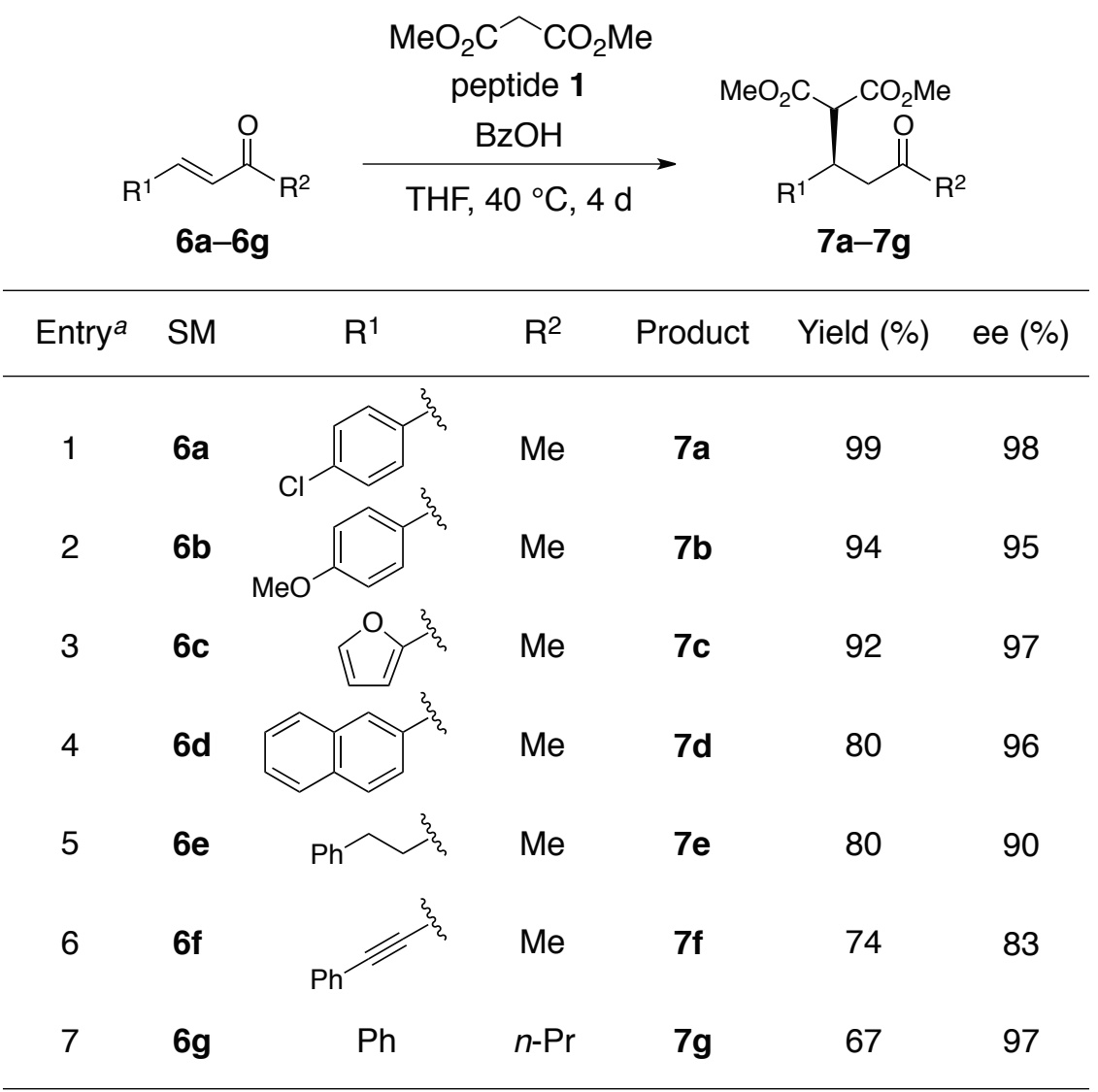

aDimethyl malonate (3 equiv), peptide 1 (20 mol\%), and $\mathrm{BzOH}(20 \mathrm{~mol} \%)$ were used in THF $(0.4 \mathrm{M})$. 
catalysis reactions (97\% ee and $96 \%$ ee, entries 3 and 4 ). When the $\mathrm{R}^{1}$ group is 2-phenylethyl or phenylethynyl group, slightly decreased enantioselectivities were observed ( $90 \%$ ee and $83 \%$ ee, entries 5 and 6). Since this is a first example of enantioselective 1,4-addition of malonate to enynone substrate, the absolute configuration of $\mathbf{7 f}$ was determined after converting to $7 \mathbf{e}$ by hydrogenation. $n$-Propyl group on the $\mathrm{R}^{2}$ position caused moderate conversion along with recovery of the starting material, though $97 \%$ ee was obtained (entry 7).

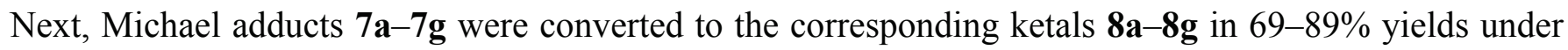
the conditions described in Table 2. When $p$-TsOH was used as an acid catalyst for the reaction of 7c, the desired product $\mathbf{8 c}$ was obtained in only $32 \%$ yield due to the partial decomposition of the desired product under the reaction conditions. Therefore, PPTS was used instead of $p$-TsOH to achieve an optimal yield (89\%, entry 3). After reduction of methyl ester moieties of $\mathbf{8 a}-\mathbf{8 g}$ by lithium aluminium hydride, the resultant diol was subjected to Kishi's reductive cyclization conditions to provide the desired 2,4,5-trisubstituted THPs $9 \mathbf{a}-\mathbf{9 g}$ in $72-87 \%$ yields with high diastereoselectivities. In the case of the reaction of $\mathbf{8 f}$ to $9 \mathbf{f}$, poor diastereomeric ratio was obtained $(\mathrm{dr}=1.4: 1$, entry 6$)$.

Table 2. Synthesis of 2,4,5-trisubstituted THPs

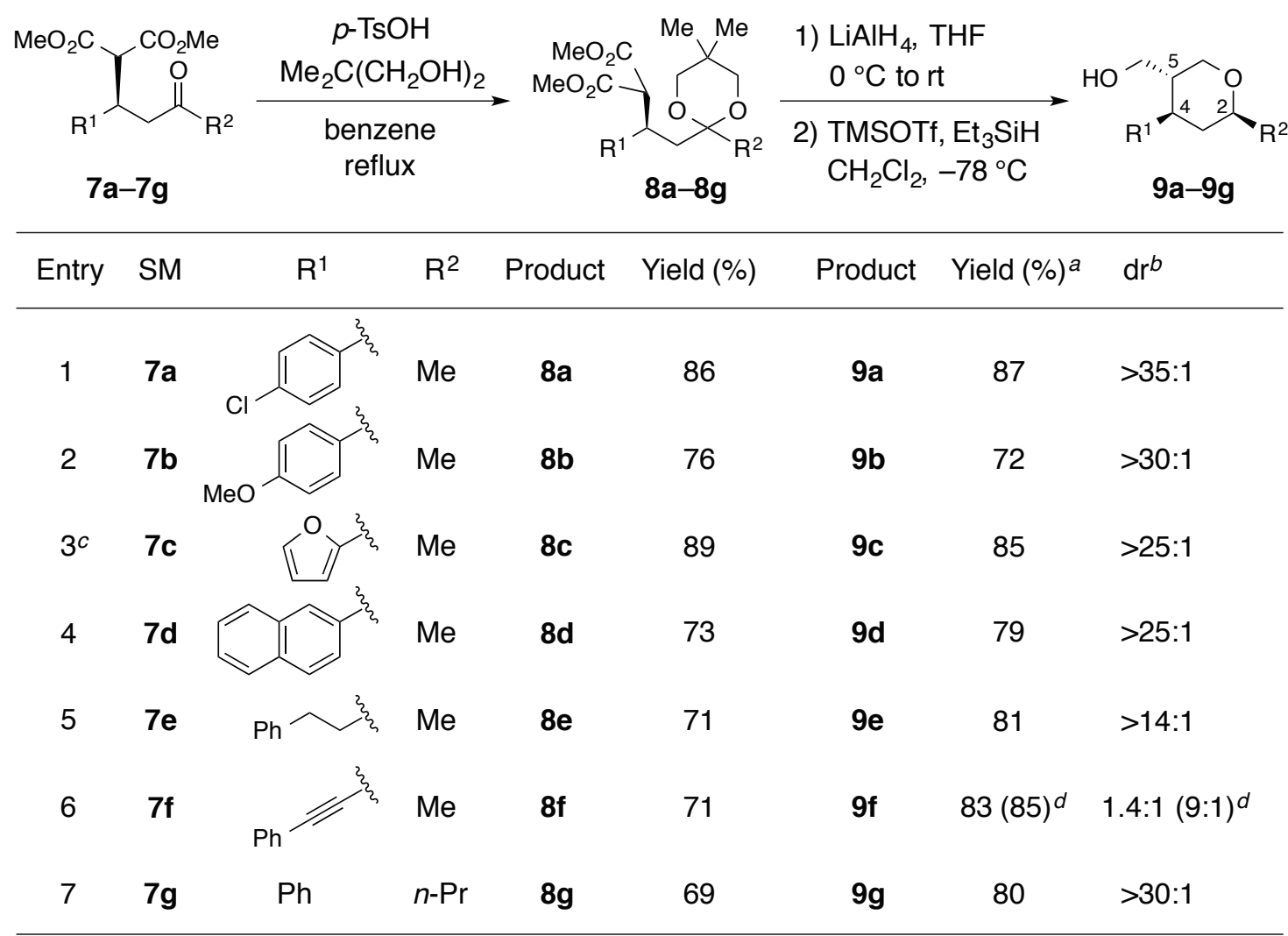

${ }^{a}$ Two-step yield. ${ }^{b}$ Determined by ${ }^{1} \mathrm{H}$ NMR. CPPTS (2 equiv) was used instead of $p$-TsOH.

${ }^{d}$ Values in brackets were obtained when triphenylsilane was used instead of triethylsilane. 
Stereochemical assignments of compounds $9 \mathbf{a}-\mathbf{9 g}$ were performed by a comparison of ${ }^{3} J$ values of axial-axial couplings on the THP rings with those of compound 5. Each ${ }^{3} J$ values of compounds $9 \mathbf{a}-\mathbf{9 g}$ showed similar values $(10.5 \sim 12.5 \mathrm{~Hz})$ to compound $\mathbf{5}$, which correspond to typical axial-axial coupling constants. Thus, three-substituent groups of compounds 9a-9g were determined as all equatorial positions. On the other hand, a side-product 9f' obtained from the reaction of $\mathbf{8 f}$ to 9 f was identified as $(2 R, 4 R, 5 S)$-diastereomer of $9 \mathbf{f}(2 S, 4 R, 5 R)$ by NOE difference experiments and coupling constants of ${ }^{1} \mathrm{H}$ NMR. ${ }^{10}$

Table 3. Selected ${ }^{3} J$ values $(\mathrm{Hz})$ of 2,4,5-trisubstituted THPs

\begin{tabular}{|c|c|c|c|c|c|c|c|c|c|c|}
\hline & 5 & $9 a$ & $9 b$ & $9 c$ & 9d & $9 e$ & $9 f$ & $9 g$ & $9 f^{\prime}$ & $\mathrm{HO}-5-6$ \\
\hline$J_{2,3 a x}$ & 10.7 & 11.0 & 10.9 & 11.2 & 10.9 & 10.9 & 11.3 & 11.5 & 11.1 & $-9 g$ \\
\hline$J_{3 a x, 4}$ & 12.2 & 12.4 & 12.3 & 12.5 & 11.8 & 11.1 & 12.2 & 11.8 & 4.5 & \\
\hline$J_{4,5}$ & 11.7 & 11.6 & 11.4 & 11.3 & 11.8 & 11.1 & 11.1 & 11.9 & 4.1 & \\
\hline$J_{5,6 a x}$ & 11.2 & 11.1 & 11.1 & 11.4 & 11.2 & 10.5 & 11.4 & 10.9 & 11.0 & \\
\hline
\end{tabular}

Scheme 2 shows a plausible reaction mechanism of the reductive cyclization of diol 8'. At first, TMSOTf promotes elimination of ketal group to give possible oxonium ions A-D with half-chair conformations. ${ }^{5}$ Diastereotopic hydroxymetyl groups were differentiated by thermodynamic preference of conformers $\mathbf{B}$ and $\mathbf{D}$ over $\mathbf{A}$ and $\mathbf{C}$ due to the pseudo-equatorial hydroxymethyl group. ${ }^{11}$ When $\mathrm{R}^{1}$ group is an aryl or an alkyl group, oxonium ion $\mathbf{B}$ where three substituents reside in pseudo-equatorial positions, is the most favorable intermediate. To the intermediate $\mathbf{B}$, nucleophilic attack of triethylsilane occurred via (b) to give a stable chair product $\mathbf{B 2}(\mathbf{9 a}-\mathbf{9 g})$ rather than the disfavorable twist-boat product B1. However, when $\mathrm{R}^{1}$ group is smaller group such as an alkyne, oxonium ion $\mathbf{D}$ could also be formed in which the alkyne substituent $\left(\mathrm{R}^{1}\right)$ resides in the pseudo-axial position. Therefore, the side-product 9f' was obtained after triethylsilane-reduction of $\mathbf{D}$ via (c) as a minor diastereomer. If that is the case, bulky silane reagents will cause a steric repulsion between pseudo-axial $\mathrm{R}^{1}$ group in conformer $\mathbf{D}$ to favor the reduction of conformer $\mathbf{B}$ via (b). In fact, when triphenylsilane was used instead of triethylsilane for the reductive cyclization of $\mathbf{8 f}$, the diastereomeric ratio was improved to 9:1 (in brackets of Table 2, entry 6).

In conclusion, we have demonstrated an enantioselective synthesis of 2,4,5-trisubstituted THP via peptide-catalyzed Michael addition reaction followed by Kishi's reductive cyclization conditions with wide substrate scope. The desired 2,4,5-trisubstituted THPs 9a-9g were obtained with 83-98\% ee and high diastereoselectivities. 


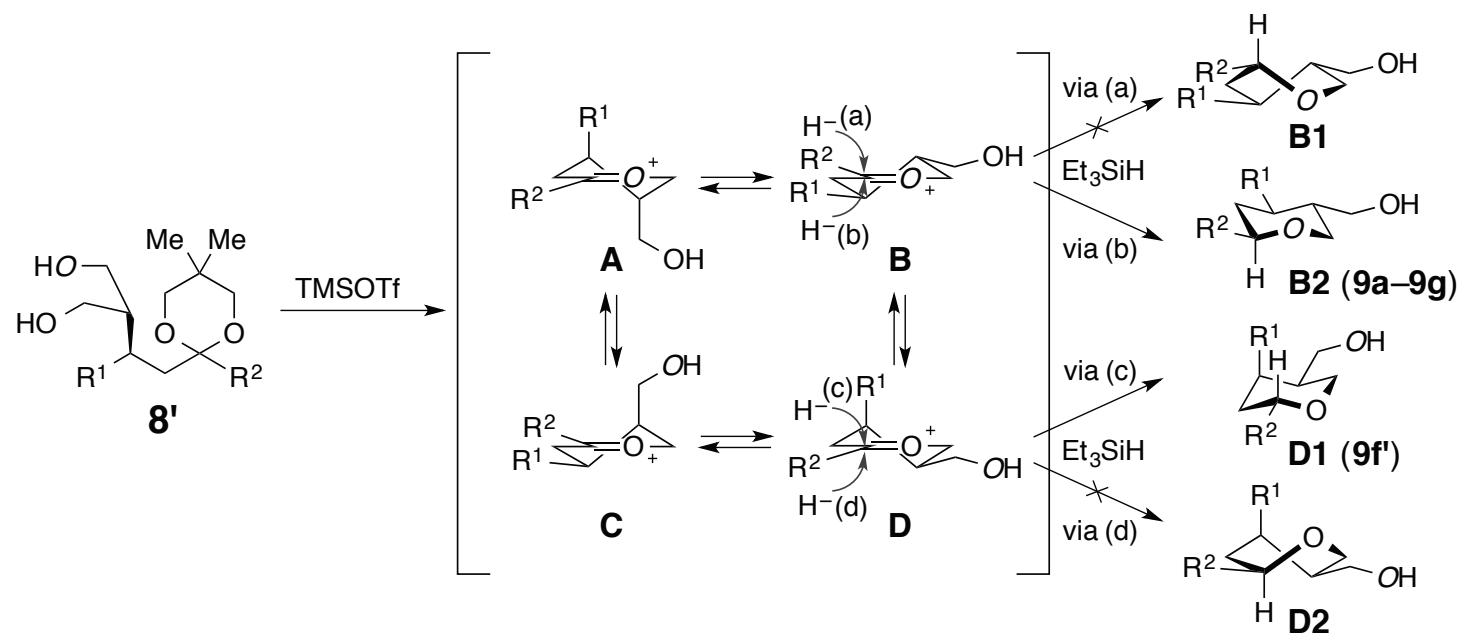

Scheme 2. Plausible mechanism of reductive cyclization

\section{EXPERIMENTAL}

General information. Specific rotations were measured on a JASCO DIP-370 polarimeter using $\mathrm{CHCl}_{3}$ as a solvent. ${ }^{1} \mathrm{H}$ NMR and ${ }^{13} \mathrm{C}$ NMR spectra were measured on Varian NMR System 500PS SN (500 MHz and $125 \mathrm{MHz}$ ) or JEOL JNM-AL-400 (400 MHz and $100 \mathrm{MHz})$. Chemical shifts $(\delta)$ are reported in parts per million (ppm) relative to the resonance of tetramethylsilane $(0.00 \mathrm{ppm})$ for ${ }^{1} \mathrm{H}$ NMR spectra, and $\mathrm{ppm}$ relative to the resonance of the central peak of $\mathrm{CDCl}_{3}(77.0 \mathrm{ppm})$ for ${ }^{13} \mathrm{C}$ NMR spectra. IR spectra were recorded on a Shimadzu IRAffinity-1 FT-IR spectrophotometer. High-resolution mass spectra (HRMS) were obtained on a JEOL JMS-T100TD using direct analysis in real time (DART) ionization in TOF mode. Silica gel (230-400 mesh) was used for flash column chromatography. Analytical thin-layer chromatography (TLC) was performed on glass pre-coated with silica gel (0.25 mm thickness). All moisture sensitive reactions were carried out under an inert atmosphere.

General procedure A: A mixture of $\alpha, \beta$-unsaturated ketone $6(0.200 \mathrm{mmol})$, dimethyl malonate $(68.5$ $\mu \mathrm{L}, 0.600 \mathrm{mmol})$, peptide $1(43.2 \mathrm{mg}, 0.0400 \mathrm{mmol})$, and benzoic acid (4.9 $\mathrm{mg}, 0.040 \mathrm{mmol})$ in THF $(0.5$ $\mathrm{mL}$ ) was heated at $40{ }^{\circ} \mathrm{C}$ for four days. The reaction mixture was diluted with $50 \%$ EtOAc in $n$-hexane and was passed through a short plug of silica gel eluted with 50\% EtOAc in $n$-hexane. After removal of solvent, the residue was purified by flash column chromatography on silica gel (eluent: EtOAc in $n$-hexane) to give the desired adduct 7 . The absolute stereochemistry was determined by comparison with literature compounds on the basis of specific rotation and HPLC chromatogram.

General procedure B: A solution of Michael adduct 7, 2,2-dimethyl-1,3-propanediol (10 equiv.), and p-toluenesulfonic acid monohydrate $(5 \mathrm{~mol} \%)$ in benzene $(0.03 \mathrm{M})$ was heated at reflux for $8 \mathrm{~h}$ using Dean-Stark apparatus. After cooling to room temperature, triethylamine (5 equiv.) was added to the 
reaction mixture, which was directly purified by flash column chromatography on silica gel (eluent: EtOAc in $n$-hexane) to give the desired ketal $\mathbf{8}$.

General procedure C: To a stirred solution of ester 8 in THF $(0.03 \mathrm{M})$ was added lithium aluminium hydride (5 equiv.) at $0{ }^{\circ} \mathrm{C}$ and the resultant white suspension was gradually warmed to room temperature overnight $(\sim 12 \mathrm{~h})$. The reaction mixture was cooled to $0{ }^{\circ} \mathrm{C}$ and was quenched by adding water, $15 \%$ $\mathrm{NaOH}$ aq., and water. The reaction mixture was warmed to room temperature and diluted with THF, then filtered through a Celite ${ }^{\circledR}$ pad (EtOAc). The filtrate was concentrated under vacuum and coevaporated with toluene twice to give crude diol 8', which was used for the next step without purification. To a solution of the above diol 8' and triethylsilane (10 equiv.) in $\mathrm{CH}_{2} \mathrm{Cl}_{2}(0.05 \mathrm{M})$ was added trimethylsilyl trifluoromethanesulfonate ( 2 equiv.) at $-78{ }^{\circ} \mathrm{C}$ and the mixture was stirred for 10 min at the same temperature. After addition of sat. $\mathrm{NaHCO}_{3}$ aq., the reaction was warmed to room temperature and extracted with $\mathrm{CHCl}_{3}$ three times. Combined organic layers were dried over anhydrous $\mathrm{MgSO}_{4}$ and concentrated under vacuum. In case TES-protected product was seen on the TLC, the crude product was treated with TBAF (2 equiv.) in THF $(0.05 \mathrm{M})$ at room temperature prior to column chromatography. The crude product was purified by flash column chromatography on silica gel (eluent: EtOAc in $n$-hexane) to give the 2,4,5-trisubstituted tetrahydropyran 9.

Dimethyl (R)-2-[1-(4-chlorophenyl)-3-oxobutyl]malonate (7a): According to the general procedure A, compound 7a was obtained from $\mathbf{6 a}$ in $99 \%$ yield. Colorless oil. Eluent for column: 20\% EtOAc in $n$-hexane. $R_{\mathrm{f}}=0.38$ (40\% EtOAc in $n$-hexane). $[\alpha]_{\mathrm{D}}^{27}-13.7\left(c 1.03, \mathrm{CHCl}_{3}\right) .{ }^{1} \mathrm{H} \mathrm{NMR}\left(500 \mathrm{MHz}, \mathrm{CDCl}_{3}\right)$

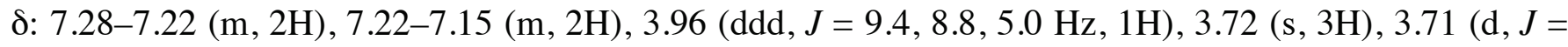
$9.5 \mathrm{~Hz}, 1 \mathrm{H}), 3.53(\mathrm{~s}, 3 \mathrm{H}), 2.97$ (dd, $J=17.2,5.0 \mathrm{~Hz}, 1 \mathrm{H}), 2.90(\mathrm{dd}, J=17.2,8.8 \mathrm{~Hz}, 1 \mathrm{H}), 2.04(\mathrm{~s}, 3 \mathrm{H})$. ${ }^{13} \mathrm{C}$ NMR (125 MHz, $\mathrm{CDCl}_{3}$ ) \&: 205.6, 168.3, 167.8, 138.9, 132.9, 129.4 (2C), 128.6 (2C), 56.7, 52.6, 52.4, 46.8, 39.6, 30.2. IR (film): 2955, 1755, $1728 \mathrm{~cm}^{-1}$. HRMS (DART) $\mathrm{m} / z:[\mathrm{M}+\mathrm{H}]^{+}$calcd for $\mathrm{C}_{15} \mathrm{H}_{18} \mathrm{ClO}_{5}, 313.0843$; found, 313.0855. HPLC (Chiralpak AD-H, 20\% $i$-propanol in $n$-hexane, flow rate $=1.0 \mathrm{~mL} / \mathrm{min}$ ): $t_{\mathrm{R}}=7.8 \min$ (major), $t_{\mathrm{R}}=9.5 \min ($ minor), ee $=98 \%$.

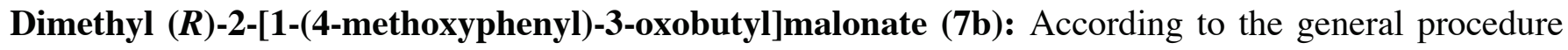
A, compound $\mathbf{7 b}$ was obtained from $\mathbf{6 b}$ in $94 \%$ yield. Colorless oil. Eluent for column: $25 \%$ EtOAc in $n$-hexane. $R_{\mathrm{f}}=0.31\left(40 \%\right.$ EtOAc in $n$-hexane). $[\alpha]_{\mathrm{D}}^{27}-16.8\left(c 1.02, \mathrm{CHCl}_{3}\right) .{ }^{1} \mathrm{H} \mathrm{NMR}\left(500 \mathrm{MHz}, \mathrm{CDCl}_{3}\right)$

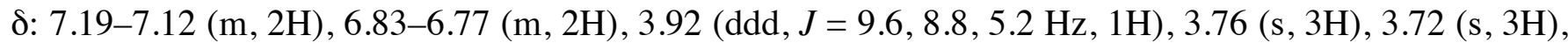
$3.69(\mathrm{~d}, J=9.6 \mathrm{~Hz}, 1 \mathrm{H}), 3.51(\mathrm{~s}, 3 \mathrm{H}), 2.94(\mathrm{dd}, J=16.7,5.2 \mathrm{~Hz}, 1 \mathrm{H}), 2.88(\mathrm{dd}, J=16.7,8.8 \mathrm{~Hz}, 1 \mathrm{H})$,

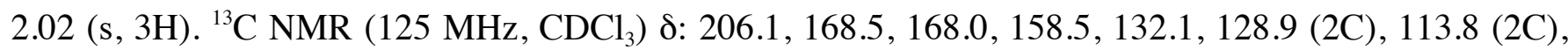


57.2, 55.0, 52.5, 52.3 , 47.2, 39.7, 30.2. IR (film): 2955, $1719 \mathrm{~cm}^{-1}$. HRMS (DART) $\mathrm{m} / z:[\mathrm{M}+\mathrm{H}]^{+}$calcd for $\mathrm{C}_{16} \mathrm{H}_{21} \mathrm{O}_{6}$, 309.1338; found, 309.1331. HPLC (Chiralpak AD-H, 20\% $i$-propanol in $n$-hexane, flow rate $=$ $1.0 \mathrm{~mL} / \mathrm{min}$ ): $t_{\mathrm{R}}=9.1 \mathrm{~min}$ (major), $t_{\mathrm{R}}=11.4 \mathrm{~min}$ (minor), ee $=95 \%$.

Dimethyl (R)-2-[1-(furan-2-yl)-3-oxobutyl]malonate (7c): According to the general procedure A, compound 7c was obtained from $\mathbf{6 c}$ in $92 \%$ yield. Colorless oil. Eluent for column: 20\% EtOAc in $n$-hexane. $R_{\mathrm{f}}=0.38\left(40 \%\right.$ EtOAc in $n$-hexane). $[\alpha]_{\mathrm{D}}^{27}-12.6\left(c 1.02, \mathrm{CHCl}_{3}\right) .{ }^{1} \mathrm{H} \mathrm{NMR}\left(500 \mathrm{MHz}, \mathrm{CDCl}_{3}\right)$

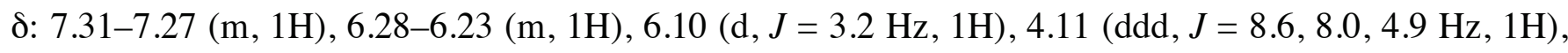
$3.82(\mathrm{~d}, J=8.0 \mathrm{~Hz}, 1 \mathrm{H}), 3.72(\mathrm{~s}, 3 \mathrm{H}), 3.64(\mathrm{~s}, 3 \mathrm{H}), 3.01(\mathrm{dd}, J=17.3,8.6 \mathrm{~Hz}, 1 \mathrm{H}), 2.93(\mathrm{dd}, J=17.3,4.9$

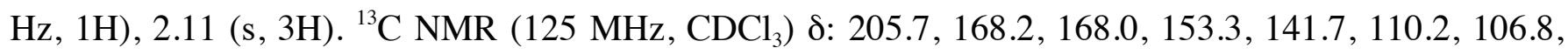
54.6, 52.6 (2C), 44.3, 33.8, 30.0. IR (film): 2957, $1732 \mathrm{~cm}^{-1}$. HRMS (DART) $\mathrm{m} / z:[\mathrm{M}+\mathrm{H}]^{+}$calcd for $\mathrm{C}_{13} \mathrm{H}_{17} \mathrm{O}_{6}, 269.1025$; found, 269.1033. HPLC (Chiralpak AD-H, 10\% EtOH in $n$-hexane, flow rate $=1.0$ $\mathrm{mL} / \mathrm{min}$, wavelength $=238 \mathrm{~nm}): t_{\mathrm{R}}=14.5 \min ($ major $), t_{\mathrm{R}}=13.1 \min ($ minor $)$, ee $=97 \%$.

Dimethyl (R)-2-[1-(naphthalen-2-yl)-3-oxobutyl]malonate (7d): According to the general procedure A, compound 7d was obtained from 6d in $80 \%$ yield. Colorless oil. Eluent for column: 30\% EtOAc in $n$-hexane. $R_{\mathrm{f}}=0.54\left(50 \%\right.$ EtOAc in $n$-hexane). $[\alpha]_{\mathrm{D}}^{28}-4.06\left(c 0.32, \mathrm{CHCl}_{3}\right) .{ }^{1} \mathrm{H} \mathrm{NMR}\left(400 \mathrm{MHz}, \mathrm{CDCl}_{3}\right)$

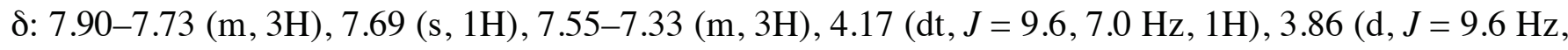
$1 \mathrm{H}), 3.73(\mathrm{~s}, 3 \mathrm{H}), 3.46(\mathrm{~s}, 3 \mathrm{H}), 3.04(\mathrm{~d}, J=7.0 \mathrm{~Hz}, 2 \mathrm{H}), 2.03(\mathrm{~s}, 3 \mathrm{H}) .{ }^{13} \mathrm{C}$ NMR $\left(100 \mathrm{MHz}, \mathrm{CDCl}_{3}\right) \delta$ : 206.1 , 168.6, 168.1 137.9, 133.3, 132.6, 128.3, 127.8, 127.6, 126.9, 126.1, 126.0, 125.9, 57.0, 52.6, 52.3, 47.0, 40.3, 30.2. IR (film): 2955, $1732 \mathrm{~cm}^{-1}$. HRMS (DART) $\mathrm{m} / z:[\mathrm{M}+\mathrm{H}]^{+}$calcd for $\mathrm{C}_{19} \mathrm{H}_{21} \mathrm{O}_{5}, 329.1389$; found, 329.1374. HPLC (Chiralpak AD-H, $10 \% i$-propanol in $n$-hexane, flow rate $=0.5 \mathrm{~mL} / \mathrm{min}$ ): $t_{\mathrm{R}}=$ $40.3 \min$ (major), $t_{\mathrm{R}}=44.4 \min$ (minor), ee $=96 \%$.

Dimethyl (R)-2-(5-oxo-1-phenylhexan-3-yl)malonate (7e): According to the general procedure A, compound 7e was obtained from $\mathbf{6 e}$ in $80 \%$ yield. Colorless oil. Eluent for column: 12\% EtOAc in $n$-hexane. $R_{\mathrm{f}}=0.46\left(40 \%\right.$ EtOAc in $n$-hexane). $[\alpha]_{\mathrm{D}}^{27}-3.53\left(c 1.02, \mathrm{CHCl}_{3}\right) .{ }^{1} \mathrm{H} \mathrm{NMR}\left(500 \mathrm{MHz}, \mathrm{CDCl}_{3}\right)$

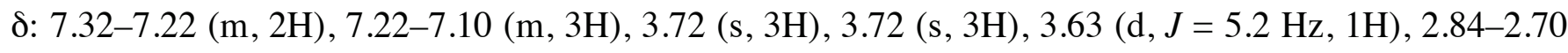
$(\mathrm{m}, 2 \mathrm{H}), 2.67-2.51(\mathrm{~m}, 3 \mathrm{H}), 2.12(\mathrm{~s}, 3 \mathrm{H}), 1.79-1.63(\mathrm{~m}, 2 \mathrm{H}) .{ }^{13} \mathrm{C} \mathrm{NMR}\left(125 \mathrm{MHz}, \mathrm{CDCl}_{3}\right) \delta: 207.3$, 169.2, 169.0, 141.4, 128.4 (2C), 128.3 (2C), 125.9, 53.6, 52.4, 52.3, 45.1, 34.1, 33.4 (2C), 30.2. IR (film): 2953, 1751, $1736 \mathrm{~cm}^{-1}$. HRMS (DART) $\mathrm{m} / z$ : $[\mathrm{M}+\mathrm{H}]^{+}$calcd for $\mathrm{C}_{17} \mathrm{H}_{23} \mathrm{O}_{5}, 307.1545$; found, 307.1544 . HPLC (Chiralpak AD-H, $5 \%$-propanol in $n$-hexane, flow rate $=0.5 \mathrm{~mL} / \mathrm{min}): t_{\mathrm{R}}=26.6$ min $($ major $), t_{\mathrm{R}}=$ $25.1 \mathrm{~min}$ (minor), ee $=90 \%$. 
Dimethyl (R)-2-(5-oxo-1-phenylhex-1-yn-3-yl)malonate (7f): According to the general procedure A, compound 7f was obtained from 6 f in 74\% yield. Colorless oil. Eluent for column: $14 \%$ EtOAc in $n$-hexane. $R_{\mathrm{f}}=0.57$ (50\% EtOAc in $n$-hexane). The absolute configuration of the major isomer was determined as $(R)$-configuratiotn after converting to compound 7e by hydrogenation $\left(\mathrm{H}_{2}, \mathrm{Pd} / \mathrm{C}, \mathrm{THF}, \mathrm{rt}\right.$, $4.5 \mathrm{~h})$ then checked by chiral HPLC analysis. $[\alpha]_{\mathrm{D}}^{27}+1.57\left(c 0.99, \mathrm{CHCl}_{3}\right) .{ }^{1} \mathrm{H} \mathrm{NMR}\left(500 \mathrm{MHz}, \mathrm{CDCl}_{3}\right)$

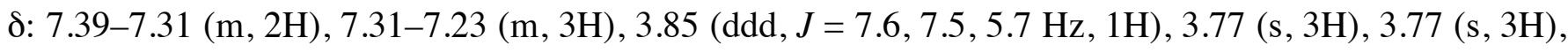
$3.74(\mathrm{~d}, J=7.6 \mathrm{~Hz}, 1 \mathrm{H}), 2.92(\mathrm{dd}, J=17.1,5.7 \mathrm{~Hz}, 1 \mathrm{H}), 2.88(\mathrm{dd}, J=17.1,7.5 \mathrm{~Hz}, 1 \mathrm{H}), 2.22(\mathrm{~s}, 3 \mathrm{H}) .{ }^{13} \mathrm{C}$ NMR (125 MHz, $\mathrm{CDCl}_{3}$ ) §: 205.3, 167.8, 167.7, 131.6 (2C), 128.11 (2C), 128.09, 122.8, 87.7, 83.2, 54.7, 52.7 (2C), 45.8, 30.2, 27.5. IR (film): 2955, $1734 \mathrm{~cm}^{-1}$. HRMS (DART) $m / z:[\mathrm{M}+\mathrm{H}]^{+}$calcd for $\mathrm{C}_{17} \mathrm{H}_{19} \mathrm{O}_{5}$, 303.1232; found, 303.1235. HPLC (Chiralpak AD-H, 5\% $i$-propanol in $n$-hexane, flow rate $=0.5$ $\mathrm{mL} / \mathrm{min}$ ): $t_{\mathrm{R}}=41.6 \min$ (major), $t_{\mathrm{R}}=35.6 \min$ (minor), ee $=83 \%$.

Dimethyl (R)-2-(3-oxo-1-phenylhexyl)malonate (7g): According to the general procedure A, compound $7 \mathrm{~g}$ was obtained from $\mathbf{6 g}$ in $67 \%$ yield. Colorless oil. Eluent for column: $12 \%$ EtOAc in $n$-hexane. $R_{\mathrm{f}}=$ 0.42 (40\% EtOAc in $n$-hexane). $[\alpha]_{\mathrm{D}}^{25}-2.95\left(c\right.$ 1.03, $\left.\mathrm{CHCl}_{3}\right) .{ }^{1} \mathrm{H}$ NMR (500 $\left.\mathrm{MHz}, \mathrm{CDCl}_{3}\right) \delta: 7.31-7.15$ $(\mathrm{m}, 5 \mathrm{H}), 3.99(\mathrm{dt}, J=9.6,7.1 \mathrm{~Hz}, 1 \mathrm{H}), 3.75(\mathrm{~d}, J=9.6 \mathrm{~Hz}, 1 \mathrm{H}), 3.72(\mathrm{~s}, 3 \mathrm{H}), 3.49$ (s, 3H), $2.91(\mathrm{~d}, J=7.1$ $\mathrm{Hz}, 2 \mathrm{H}), 2.28(\mathrm{dt}, J=16.8,7.3 \mathrm{~Hz}, 1 \mathrm{H}), 2.21(\mathrm{dt}, J=16.8,7.2 \mathrm{~Hz}, 1 \mathrm{H}), 1.47$ (qdd, $J=7.4,7.3,7.2 \mathrm{~Hz}$,

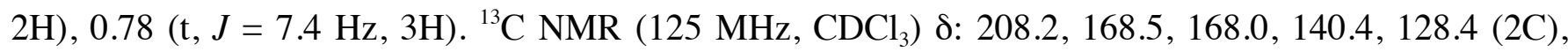
127.9 (2C), 127.1, 57.1, 52.5, 52.3, 46.2, 45.0, 40.4, 16.9, 13.5. IR (film): 2959, 1738, $1717 \mathrm{~cm}^{-1}$. HRMS (DART) $m / z:[\mathrm{M}+\mathrm{H}]^{+}$calcd for $\mathrm{C}_{17} \mathrm{H}_{23} \mathrm{O}_{5}, 307.1546$; found, 307.1549. HPLC (Chiralpak AD-H, 10\% $i$-propanol in $n$-hexane, flow rate $=1 \mathrm{~mL} / \mathrm{min}$ ): $t_{\mathrm{R}}=11.0 \mathrm{~min}$ (major), $t_{\mathrm{R}}=13.5 \mathrm{~min}$ (minor), ee $=97 \%$.

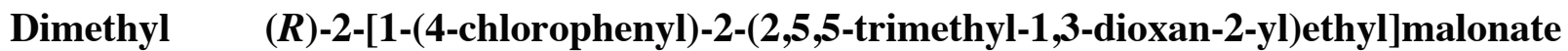

(8a):

According to the general procedure B, compound 8a was obtained from 7a in $86 \%$ yield. Colorless oil. Eluent for column: $15 \%$ EtOAc in $n$-hexane. $R_{\mathrm{f}}=0.43\left(30 \%\right.$ EtOAc in $n$-hexane). $[\alpha]_{\mathrm{D}}^{26}+0.72(c 1.00$,

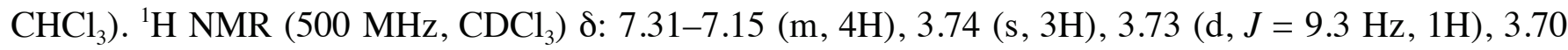
(ddd, $J=9.3,8.5,3.0 \mathrm{~Hz}, 1 \mathrm{H}), 3.47(\mathrm{~s}, 3 \mathrm{H}), 3.45(\mathrm{~s}, 2 \mathrm{H}), 3.31(\mathrm{~d}, J=11.4 \mathrm{~Hz}, 1 \mathrm{H}), 3.22(\mathrm{~d}, J=11.4 \mathrm{~Hz}$, $1 \mathrm{H}), 2.22(\mathrm{dd}, J=14.4,3.2 \mathrm{~Hz}, 1 \mathrm{H}), 2.13(\mathrm{dd}, J=14.4,8.5 \mathrm{~Hz}, 1 \mathrm{H}), 1.15$ (s, 3H), 0.92 (s, 3H), 0.89 (s, 3H). ${ }^{13} \mathrm{C}$ NMR (125 MHz, $\mathrm{CDCl}_{3}$ ) $\delta: 168.6,168.0,140.4,132.5,129.9$ (2C), 128.3 (2C), 98.5, 70.3, 70.1, 58.5, 52.5, 52.2 , 40.3 , 40.1 , 29.7, 22.8, 22.5, 22.4. IR (film): 2955, 2870, $1732 \mathrm{~cm}^{-1}$. HRMS (DART) $\mathrm{m} / z$ : $[\mathrm{M}+\mathrm{H}]^{+}$calcd for $\mathrm{C}_{20} \mathrm{H}_{28} \mathrm{ClO}_{6}, 399.1573$; found, 399.1574 . 
Eluent for column: $15 \%$ EtOAc in $n$-hexane. $R_{\mathrm{f}}=0.40$ (30\% EtOAc in $n$-hexane). $[\alpha]_{\mathrm{D}}^{26}-6.60(c 1.00$,

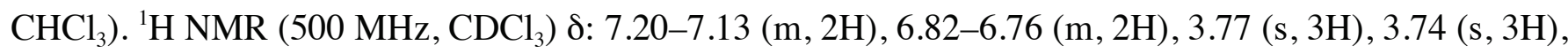
$3.71(\mathrm{~d}, J=9.8 \mathrm{~Hz}, 1 \mathrm{H}), 3.62(\mathrm{ddd}, J=9.8,9.6,3.2 \mathrm{~Hz}, 1 \mathrm{H}), 3.53(\mathrm{~d}, J=11.4 \mathrm{~Hz}, 1 \mathrm{H}), 3.45(\mathrm{~s}, 3 \mathrm{H}), 3.43$ $(\mathrm{d}, J=11.4 \mathrm{~Hz}, 1 \mathrm{H}), 3.26(\mathrm{~s}, 2 \mathrm{H}), 2.24(\mathrm{dd}, J=14.4,3.2 \mathrm{~Hz}, 1 \mathrm{H}), 2.11(\mathrm{dd}, J=14.4,9.6 \mathrm{~Hz}, 1 \mathrm{H}), 1.10$ (s, $3 \mathrm{H}), 0.93$ (s, 3H), 0.88 (s, 3H). ${ }^{13} \mathrm{C} \mathrm{NMR}\left(125 \mathrm{MHz} \mathrm{CDCl}_{3}\right)$ \&: 168.9, 168.2, 158.3, 133.5, 129.4 (2C), 113.5 (2C), 98.6, 70.3, 70.0, 58.9, 55.1, 52.4, 52.1, 40.3, 39.2, 29.7, 23.2, 22.63, 22.59. IR (film): 2955, 2870, $1734 \mathrm{~cm}^{-1}$. HRMS (DART) $\mathrm{m} / z:[\mathrm{M}+\mathrm{H}]^{+}$calcd for $\mathrm{C}_{21} \mathrm{H}_{31} \mathrm{O}_{7}, 395.2070$; found, 395.2052 .

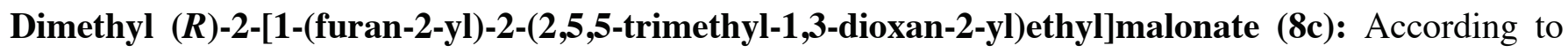
the general procedure $\mathrm{B}$, except using PPTS (2 equiv.) instead of $p$-TsOH, compound 8c was obtained from $7 \mathrm{c}$ in $89 \%$ yield. Colorless oil. Eluent for column: $10 \%$ EtOAc in $n$-hexane. $R_{\mathrm{f}}=0.43(30 \%$ EtOAc

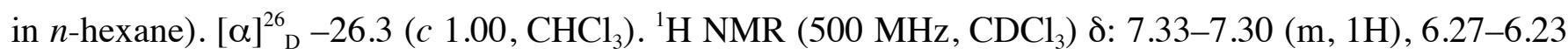
(m, 1H), 6.13-6.09 (m, 1H), 3.84-3.81 (m, 1H), $3.78(\mathrm{~d}, J=9.4 \mathrm{~Hz}, 1 \mathrm{H}), 3.75(\mathrm{~s}, 3 \mathrm{H}), 3.57(\mathrm{~s}, 3 \mathrm{H}), 3.51$ $(\mathrm{d}, J=11.4 \mathrm{~Hz}, 1 \mathrm{H}), 3.46(\mathrm{~d}, J=11.4 \mathrm{~Hz}, 1 \mathrm{H}), 3.37(\mathrm{~d}, J=11.5 \mathrm{~Hz}, 1 \mathrm{H}), 3.34(\mathrm{~d}, J=11.5 \mathrm{~Hz}, 1 \mathrm{H})$,

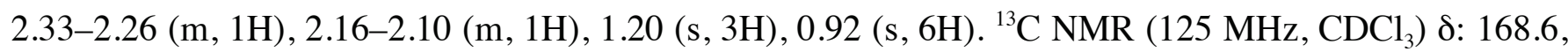
168.2, 154.2, 141.2, 110.2, 107.2, 98.3, 70.3, 70.2, 56.5, 52.5, 52.4, 37.2, 34.6, 29.8, 22.7, 22.6, 22.0. IR (film): 2955, 2870, $1736 \mathrm{~cm}^{-1}$. HRMS (DART) $\mathrm{m} / \mathrm{z}:[\mathrm{M}+\mathrm{H}]^{+}$calcd for $\mathrm{C}_{18} \mathrm{H}_{27} \mathrm{O}_{7}, 355.1757$; found, 355.1772 .

\section{Dimethyl $\quad(R)-2-[1-(n a p h t h a l e n-2-y l)-2-(2,5,5-t r i m e t h y l-1,3-d i o x a n-2-y l) e t h y l] m a l o n a t e \quad(8 d):$} According to the general procedure B, compound $\mathbf{8 d}$ was obtained from $\mathbf{7 d}$ in $73 \%$ yield. Colorless oil. Eluent for column: $20 \% \mathrm{EtOAc}$ in $n$-hexane. $R_{\mathrm{f}}=0.31\left(30 \%\right.$ EtOAc in $n$-hexane). $[\alpha]^{26}+3.71(c 1.03$, $\left.\mathrm{CHCl}_{3}\right) .{ }^{1} \mathrm{H} \mathrm{NMR}\left(500 \mathrm{MHz}, \mathrm{CDCl}_{3}\right)$ 8: 7.80-7.74 (m, 3H), $7.71(\mathrm{~d}, J=1.7 \mathrm{~Hz}, 1 \mathrm{H}), 7.48-7.39$ (m, 3H), $3.88-3.83(\mathrm{~m}, 2 \mathrm{H}), 3.76(\mathrm{~s}, 3 \mathrm{H}), 3.52(\mathrm{~d}, J=11.3 \mathrm{~Hz}, 1 \mathrm{H}), 3.45(\mathrm{~d}, J=11.3 \mathrm{~Hz}, 1 \mathrm{H}), 3.37(\mathrm{~s}, 3 \mathrm{H}), 3.24(\mathrm{~s}$, 2H), 2.37-2.25 (m, 2H), $1.11(\mathrm{~s}, 3 \mathrm{H}), 0.90$ (s, 6H). ${ }^{13} \mathrm{C}$ NMR $\left(125 \mathrm{MHz}, \mathrm{CDCl}_{3}\right) \delta: 168.8,168.1,139.2$, 133.2, 132.4, 127.80, 127.76, 127.51, 127.46, 126.5, 125.8, 125.5, 98.6, 70.3 , 70.1, 58.8, 52.5, 52.2, 41.1, 39.4, 29.7, 22.9, 22.7, 22.6. IR (film): 3019, 2957, $1734 \mathrm{~cm}^{-1}$. HRMS (DART) $\mathrm{m} / z:[\mathrm{M}+\mathrm{H}]^{+}$calcd for $\mathrm{C}_{24} \mathrm{H}_{31} \mathrm{O}_{6}, 415.2121$; found, 415.2129 .

Dimethyl $(R)$-2-[4-phenyl-1-(2,5,5-trimethyl-1,3-dioxan-2-yl)butan-2-yl]malonate (8e): According to the general procedure B, compound $\mathbf{8 e}$ was obtained from $\mathbf{7 e}$ in $71 \%$ yield. Colorless oil. Eluent for column: $10 \%$ EtOAc in $n$-hexane. $R_{\mathrm{f}}=0.51$ (30\% EtOAc in $n$-hexane). $[\alpha]^{26}-14.3\left(c 1.00, \mathrm{CHCl}_{3}\right) .{ }^{1} \mathrm{H}$ NMR (500 MHz, $\left.\mathrm{CDCl}_{3}\right)$ 8: 7.31-7.21 (m, 2H), 7.21-7.12 (m, 3H), 3.99 (d, J=4.4 Hz, 1H), $3.71(\mathrm{~s}, 3 \mathrm{H})$, 
$3.65(\mathrm{~s}, 3 \mathrm{H}), 3.60(\mathrm{dd}, J=11.1,3.0 \mathrm{~Hz}, 2 \mathrm{H}), 3.42-3.34(\mathrm{~m}, 2 \mathrm{H}), 2.67(\mathrm{ddd}, J=13.7,9.7,5.9 \mathrm{~Hz}, 1 \mathrm{H})$, 2.63-2.52 (m, 2H), $1.97(\mathrm{dd}, J=14.8,7.2 \mathrm{~Hz}, 1 \mathrm{H}), 1.87-1.73(\mathrm{~m}, 3 \mathrm{H}), 1.38(\mathrm{~s}, 3 \mathrm{H}), 1.05$ (s, 3H), 0.80 (s, $3 \mathrm{H}) .{ }^{13} \mathrm{C} \mathrm{NMR}\left(125 \mathrm{MHz}, \mathrm{CDCl}_{3}\right.$ ) $\delta: 169.9,169.6,142.2,128.5$ (2C), 128.2 (2C), 125.6, 99.2, 70.3 (2C), 54.7, 52.1 , 52.0, 41.0, 34.3, 33.6, 33.3, 29.8, 22.9, 22.4, 19.3. IR (film): 2963, 2868, $1732 \mathrm{~cm}^{-1}$. HRMS (DART) $m / z:[\mathrm{M}+\mathrm{H}]^{+}$calcd for $\mathrm{C}_{22} \mathrm{H}_{33} \mathrm{O}_{6}, 393.2277$; found, 393.2280.

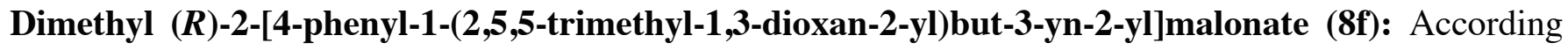
to the general procedure B, compound $8 \mathbf{f}$ was obtained from $7 \mathbf{f}$ in $71 \%$ yield. Colorless oil. Eluent for column: $20 \%$ EtOAc in $n$-hexane. $R_{\mathrm{f}}=0.43$ (30\% EtOAc in $n$-hexane). $[\alpha]^{26}{ }_{\mathrm{D}}+11.0\left(c 1.02, \mathrm{CHCl}_{3}\right) .{ }^{1} \mathrm{H}$ NMR (500 MHz, $\left.\mathrm{CDCl}_{3}\right)$ d: 7.38-7.32 (m, 2H), 7.29-7.23 (m, 3H), $3.81(\mathrm{~d}, J=7.5 \mathrm{~Hz}, 1 \mathrm{H}), 3.78(\mathrm{~s}, 6 \mathrm{H})$, $3.68(\mathrm{ddd}, J=7.5,7.1,5.6 \mathrm{~Hz}, 1 \mathrm{H}), 3.55(\mathrm{~d}, J=11.4 \mathrm{~Hz}, 2 \mathrm{H}), 3.53-3.49$ (m, 2H), 2.14 (dd, $J=14.3,7.1$ $\mathrm{Hz}, 1 \mathrm{H}), 2.11(\mathrm{dd}, J=14.3,5.6 \mathrm{~Hz}, 1 \mathrm{H}), 1.53(\mathrm{~s}, 3 \mathrm{H}), 1.01(\mathrm{~s}, 3 \mathrm{H}), 0.93$ (s, 3H). ${ }^{13} \mathrm{C} \mathrm{NMR}(125 \mathrm{MHz}$,

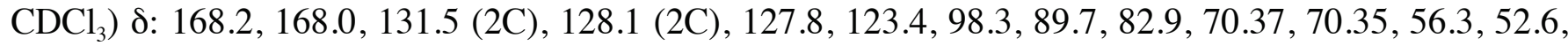
52.5, 39.9, 29.9, 27.6, 22.8, 22.5, 21.4. IR (film): 3021, $1736 \mathrm{~cm}^{-1}$. HRMS (DART) $\mathrm{m} / z:[\mathrm{M}+\mathrm{H}]^{+}$calcd for $\mathrm{C}_{22} \mathrm{H}_{29} \mathrm{O}_{6}, 389.1964$; found, 389.1980 .

Dimethyl (R)-2-[2-(5,5-dimethyl-2-propyl-1,3-dioxan-2-yl)-1-phenylethyl]malonate (8g): According to the general procedure B, compound $\mathbf{8 g}$ was obtained from $\mathbf{7 g}$ in $69 \%$ yield. Yellow oil. Eluent for column: $20 \%$ EtOAc in $n$-hexane. $R_{\mathrm{f}}=0.51$ (30\% EtOAc in $n$-hexane). $[\alpha]^{26}+3.74\left(c 1.02, \mathrm{CHCl}_{3}\right) .{ }^{1} \mathrm{H}$

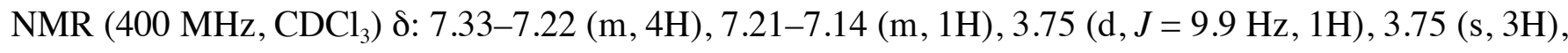
$3.64(\mathrm{ddd}, J=9.9,7.8,4.7 \mathrm{~Hz}, 1 \mathrm{H}), 3.55(\mathrm{~d}, J=11.2 \mathrm{~Hz}, 1 \mathrm{H}), 3.42(\mathrm{~d}, J=11.2 \mathrm{~Hz}, 1 \mathrm{H}), 3.42(\mathrm{~s}, 3 \mathrm{H})$, $3.20(\mathrm{~d}, J=11.4 \mathrm{~Hz}, 1 \mathrm{H}), 3.15(\mathrm{~d}, J=11.4 \mathrm{~Hz}, 1 \mathrm{H}), 2.20(\mathrm{dd}, J=14.6,7.8 \mathrm{~Hz}, 1 \mathrm{H}), 2.15(\mathrm{dd}, J=14.6$, $4.7 \mathrm{~Hz}, 1 \mathrm{H}), 1.55-1.46(\mathrm{~m}, 1 \mathrm{H}), 1.33-1.14(\mathrm{~m}, 3 \mathrm{H}), 0.93(\mathrm{~s}, 3 \mathrm{H}), 0.85(\mathrm{~s}, 3 \mathrm{H}), 0.71(\mathrm{t}, J=6.9 \mathrm{~Hz}, 3 \mathrm{H})$.

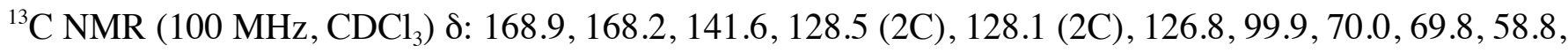
52.4, 52.1, 40.9, 37.5, 36.0, 29.5, 22.63, 22.61, 16.1, 14.0. IR (film): 3021, 2969, $1736 \mathrm{~cm}^{-1}$. HRMS (DART) $m / z:[\mathrm{M}+\mathrm{H}]^{+}$calcd for $\mathrm{C}_{22} \mathrm{H}_{33} \mathrm{O}_{6}, 393.2277$; found, 393.2294 .

(2S,4R,5R)-4-(4-Chlorophenyl)-5-hydroxymethyl-2-methyltetrahydropyran (9a): According to the general procedure C, compound 9a was obtained from 8a in 87\% yield (2 steps). White solid. Eluent for column: $15 \%$ EtOAc in $n$-hexane. $R_{\mathrm{f}}=0.23\left(40 \%\right.$ EtOAc in $n$-hexane). Mp $69-72{ }^{\circ} \mathrm{C} .[\alpha]^{27}{ }_{\mathrm{D}}-21.2(c 1.00$,

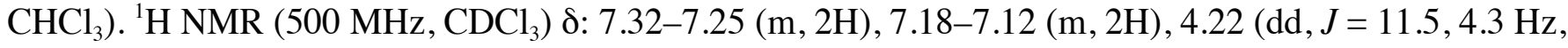
$1 \mathrm{H}), 3.54(\mathrm{dqd}, J=11.0,6.3,2.1 \mathrm{~Hz}, 1 \mathrm{H}), 3.45(\mathrm{dd}, J=11.5,11.1 \mathrm{~Hz}, 1 \mathrm{H}), 3.43(\mathrm{dd}, J=11.0,3.2 \mathrm{~Hz}$, $1 \mathrm{H}), 3.26(\mathrm{dd}, J=11.0,6.9 \mathrm{~Hz}, 1 \mathrm{H}), 2.64(\mathrm{ddd}, J=12.4,11.6,4.0 \mathrm{~Hz}, 1 \mathrm{H}), 1.95(\mathrm{ddddd}, J=11.6,11.1$, 
6.8, 4.3, 3.2 Hz, 1H), $1.76(\mathrm{ddd}, J=13.3,4.0,2.1 \mathrm{~Hz}, 1 \mathrm{H}), 1.48(\mathrm{ddd}, J=13.3,12.4,11.0 \mathrm{~Hz}, 1 \mathrm{H}), 1.22$ $(\mathrm{d}, J=6.3 \mathrm{~Hz}, 3 \mathrm{H}), 1.11$ (br s, $1 \mathrm{H}) .{ }^{13} \mathrm{C} \mathrm{NMR}\left(125 \mathrm{MHz}, \mathrm{CDCl}_{3}\right) \delta: 142.2,132.2,128.8(2 \mathrm{C}), 128.7$ (2C), 73.8, 70.7, 62.1 43.5, 43.4, 41.7, 21.7. IR (KBr): 3422, $2928 \mathrm{~cm}^{-1}$. HRMS (DART) m/z: [M+H] calcd for $\mathrm{C}_{13} \mathrm{H}_{18} \mathrm{ClO}_{2}, 241.0995$; found, 241.0997.

(2S,4R,5R)-5-Hydroxymethyl-4-(4-methoxyphenyl)-2-methyltetrahydropyran (9b): According to the general procedure $\mathrm{C}$, compound $\mathbf{9 b}$ was obtained from $\mathbf{8 b}$ in $72 \%$ yield (2 steps). White solid. Eluent for column: $20 \%$ EtOAc in $n$-hexane. $R_{\mathrm{f}}=0.20$ (40\% EtOAc in $n$-hexane). Mp 86-87 ${ }^{\circ} \mathrm{C}$. $[\alpha]^{27}{ }_{\mathrm{D}}-18.6(c 1.00$,

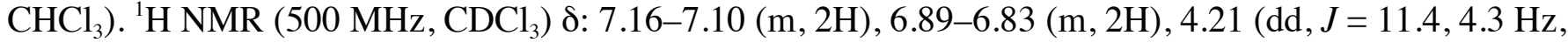
$1 \mathrm{H}), 3.79(\mathrm{~s}, 3 \mathrm{H}), 3.54(\mathrm{dqd}, J=10.9,6.2,2.1 \mathrm{~Hz}, 1 \mathrm{H}), 3.43(\mathrm{dd}, J=11.0,3.2 \mathrm{~Hz}, 1 \mathrm{H}), 3.42(\mathrm{dd}, J=11.4$, $11.1 \mathrm{~Hz}, 1 \mathrm{H}), 3.26(\mathrm{dd}, J=11.0,6.9 \mathrm{~Hz}, 1 \mathrm{H}), 2.56(\mathrm{ddd}, J=12.3,11.4,4.0 \mathrm{~Hz}, 1 \mathrm{H}), 1.94$ (dddd, $J=$ $11.4,11.1,6.9,4.3,3.2 \mathrm{~Hz}, 1 \mathrm{H}), 1.76$ (ddd, $J=13.3,4.0,2.1 \mathrm{~Hz}, 1 \mathrm{H}), 1.49$ (ddd, $J=13.3,12.3,10.9 \mathrm{~Hz}$,

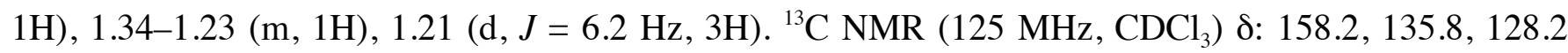
(2C), 114.1 (2C), 73.9, 70.9, 62.4, 55.2, 43.8, 43.4, 42.0, 21.7. IR (KBr): 3422, $2916 \mathrm{~cm}^{-1}$. HRMS (DART) $m / z:[\mathrm{M}+\mathrm{H}]^{+}$calcd for $\mathrm{C}_{14} \mathrm{H}_{21} \mathrm{O}_{3}, 237.1491$; found, 237.1492.

(2S,4R,5R)-4-(2-Furyl)-5-hydroxymethyl-2-methyltetrahydropyran (9c): According to the general procedure $\mathrm{C}$, compound 9c was obtained from $8 \mathbf{c}$ in $85 \%$ yield (2 steps). Colorless oil. Eluent for column: $15 \%$ EtOAc in $n$-hexane. $R_{\mathrm{f}}=0.24$ (40\% EtOAc in $n$-hexane). $[\alpha]^{27}{ }_{\mathrm{D}}-7.06\left(c 1.00, \mathrm{CHCl}_{3}\right) .{ }^{1} \mathrm{H}$ NMR

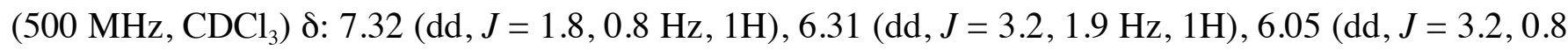
$\mathrm{Hz}, 1 \mathrm{H}), 4.15(\mathrm{dd}, J=11.5,4.3 \mathrm{~Hz}, 1 \mathrm{H}), 3.55(\mathrm{dd}, J=11.3,3.3 \mathrm{~Hz}, 1 \mathrm{H}), 3.51(\mathrm{dqd}, J=11.2,6.2,2.1 \mathrm{~Hz}$, $1 \mathrm{H}), 3.44(\mathrm{dd}, J=11.3,5.8 \mathrm{~Hz}, 1 \mathrm{H}), 3.43(\mathrm{dd}, J=11.5,11.4 \mathrm{~Hz}, 1 \mathrm{H}), 2.82(\mathrm{ddd}, J=12.5,11.3,4.0 \mathrm{~Hz}$, 1H), 1.92 (ddddd, $J=11.4,11.3,5.8,4.3,3.3 \mathrm{~Hz}, 1 \mathrm{H}$ ), 1.84 (ddd, $J=13.2,4.0,2.1 \mathrm{~Hz}, 1 \mathrm{H}$ ), 1.59 (ddd, $J$ $=13.2,12.5,11.2 \mathrm{~Hz}, 1 \mathrm{H}), 1.34-1.25(\mathrm{~m}, 1 \mathrm{H}), 1.23(\mathrm{~d}, J=6.2 \mathrm{~Hz}, 3 \mathrm{H}) .{ }^{13} \mathrm{C} \mathrm{NMR}\left(125 \mathrm{MHz}, \mathrm{CDCl}_{3}\right) \delta$ : 157.3, 141.0, 110.1, 104.8, 73.4, 70.5, 62.5, 42.9, 38.7, 36.9, 21.7. IR (KBr): 3449, $2920 \mathrm{~cm}^{-1}$. HRMS (DART) $m / z:[\mathrm{M}+\mathrm{H}]^{+}$calcd for $\mathrm{C}_{11} \mathrm{H}_{17} \mathrm{O}_{3}, 197.1178$; found, 197.1181 .

(2S,4R,5R)-5-Hydroxymethyl-2-methyl-4-(2-naphthyl)tetrahydropyran (9d): According to the general procedure C, compound 9d was obtained from 8d in 79\% yield (2 steps). Colorless oil. Eluent for column: $18 \%$ EtOAc in $n$-hexane. $R_{\mathrm{f}}=0.16$ (30\% EtOAc in $n$-hexane). $[\alpha]^{25}{ }_{\mathrm{D}}-21.4\left(c 1.00, \mathrm{CHCl}_{3}\right) .{ }^{1} \mathrm{H}$ NMR (400 MHz, $\left.\mathrm{CDCl}_{3}\right)$ ঠ: 7.96-7.75 (m, 3H), 7.73-7.63 (m, 1H), 7.55-7.42 (m, 2H), 7.37 (dd, $J=8.6$, $1.9 \mathrm{~Hz}, 1 \mathrm{H}), 4.27(\mathrm{dd}, J=11.4,4.4 \mathrm{~Hz}, 1 \mathrm{H}), 3.60(\mathrm{dqd}, J=10.9,6.3,2.0 \mathrm{~Hz}, 1 \mathrm{H}), 3.50(\mathrm{dd}, J=11.4,11.2$ $\mathrm{Hz}, 1 \mathrm{H}), 3.45(\mathrm{dd}, J=11.1,3.4 \mathrm{~Hz}, 1 \mathrm{H}), 3.29(\mathrm{dd}, J=11.1,6.9 \mathrm{~Hz}, 1 \mathrm{H}), 2.80(\mathrm{td}, J=11.8,4.1 \mathrm{~Hz}, 1 \mathrm{H})$, 
2.12 (ddddd, $J=11.8,11.2,6.9,4.4,3.4 \mathrm{~Hz}, 1 \mathrm{H}), 1.84(\mathrm{ddd}, J=13.2,4.1,2.0 \mathrm{~Hz}, 1 \mathrm{H}), 1.64(\mathrm{ddd}, J=$ 13.2, 11.8, $10.9 \mathrm{~Hz}, 1 \mathrm{H}), 1.25(\mathrm{~d}, J=6.3 \mathrm{~Hz}, 3 \mathrm{H}) .{ }^{13} \mathrm{C} \mathrm{NMR}\left(100 \mathrm{MHz}, \mathrm{CDCl}_{3}\right)$ 8: 141.3, 133.7, 132.5, $128.5,127.7,127.6,126.2$, 126.0, 125.6, 125.5, 73.9, 70.9, 62.4, 44.3, 43.4, 41.7, 21.7. IR (KBr): 3399, 2968, $2928 \mathrm{~cm}^{-1}$. HRMS (DART) $\mathrm{m} / z:[\mathrm{M}+\mathrm{H}]^{+}$calcd for $\mathrm{C}_{17} \mathrm{H}_{21} \mathrm{O}_{2}, 257.1542$; found, 257.1554 .

(2S,4R,5R)-5-Hydroxymethyl-2-methyl-4-(2-phenylethyl)tetrahydropyran (9e): According to the general procedure $\mathrm{C}$, compound $\mathbf{9 e}$ was obtained from $\mathbf{8 e}$ in $81 \%$ yield (2 steps). Colorless oil. Eluent for column: $15 \%$ EtOAc in $n$-hexane. $R_{\mathrm{f}}=0.24$ (40\% EtOAc in $n$-hexane). $[\alpha]^{27}{ }_{\mathrm{D}}-52.6\left(c 1.00, \mathrm{CHCl}_{3}\right) .{ }^{1} \mathrm{H}$ NMR (500 MHz, $\left.\mathrm{CDCl}_{3}\right)$ ঠ: 7.31-7.25 (m, 2H), 7.22-7.15 (m, 3H), $4.08(\mathrm{dd}, J=11.4,3.5 \mathrm{~Hz}, 1 \mathrm{H}), 3.72$ $(\mathrm{dd}, J=11.1,2.3 \mathrm{~Hz}, 1 \mathrm{H}), 3.52(\mathrm{dd}, J=11.1,5.9 \mathrm{~Hz}, 1 \mathrm{H}), 3.41(\mathrm{dqd}, J=10.9,6.2,2.0 \mathrm{~Hz}, 1 \mathrm{H}), 3.30(\mathrm{dd}$, $J=11.4,10.5 \mathrm{~Hz}, 1 \mathrm{H}), 2.74(\mathrm{ddd}, J=13.5,10.7,4.9 \mathrm{~Hz}, 1 \mathrm{H}), 2.49(\mathrm{ddd}, J=13.5,10.2,6.3 \mathrm{~Hz}, 1 \mathrm{H}), 1.92$ (ddddd, $J=11.1,10.5,5.9,3.5,2.3 \mathrm{~Hz}, 1 \mathrm{H}), 1.85$ (ddd, $J=13.1,2.6,2.6 \mathrm{~Hz}, 1 \mathrm{H}), 1.70-1.48(\mathrm{~m}, 3 \mathrm{H})$, $1.48-1.39(\mathrm{~m}, 1 \mathrm{H}), 1.21(\mathrm{~d}, J=6.2 \mathrm{~Hz}, 3 \mathrm{H}), 1.07(\mathrm{ddd}, J=13.1,11.1,10.9 \mathrm{~Hz}, 1 \mathrm{H}) .{ }^{13} \mathrm{C} \mathrm{NMR}(125 \mathrm{MHz}$, $\left.\mathrm{CDCl}_{3}\right) \delta: 142.5,128.4(2 \mathrm{C}), 128.2(2 \mathrm{C}), 125.8,73.6,70.6,62.0,42.9,38.7,35.6,34.9,32.3,22.0 . \mathrm{IR}$ (KBr): 3443, $2928 \mathrm{~cm}^{-1}$. HRMS (DART) $\mathrm{m} / z:[\mathrm{M}+\mathrm{H}]^{+}$calcd for $\mathrm{C}_{15} \mathrm{H}_{23} \mathrm{O}_{2}, 235.1698$; found, 235.1693 .

$(2 S, 4 R, 5 R)$ - and $(2 R, 4 R, 5 S)-5$-Hydroxymethyl-2-methyl-4-(2-phenylethynyl)tetrahydropyran (9f and 9f'): According to the general procedure C, compounds 9f and 9f' were obtained from 8f in $49 \%$ and $34 \%$ yields ( 2 steps), respectively. When the reaction was performed using triphenylsilane (10 equiv.) instead of triethylsilane, compounds 9f and 9f' were obtained in 85\% in 2 steps with 9:1 dr. Colorless oil. Eluent for column: $16 \%$ (for 9f) and 20\% (for 9f') EtOAc in $n$-hexane. 9f: $R_{\mathrm{f}}=0.19$ (30\% EtOAc in $n$-hexane). $[\alpha]^{26}-0.40\left(c 1.00, \mathrm{CHCl}_{3}\right) .{ }^{1} \mathrm{H} \mathrm{NMR}\left(500 \mathrm{MHz}, \mathrm{CDCl}_{3}\right)$ 8: 7.43-7.35 (m, 2H), 7.32-7.27 (m, $3 \mathrm{H}), 4.11(\mathrm{dd}, J=11.5,4.3 \mathrm{~Hz}, 1 \mathrm{H}), 3.90(\mathrm{dd}, J=11.1,3.7 \mathrm{~Hz}, 1 \mathrm{H}), 3.71(\mathrm{dd}, J=11.1,6.3 \mathrm{~Hz}, 1 \mathrm{H}), 3.43$ $(\mathrm{dqd}, J=11.3,6.2,1.9 \mathrm{~Hz}, 1 \mathrm{H}), 3.33(\mathrm{t}, J=11.4 \mathrm{~Hz}, 1 \mathrm{H}), 2.61(\mathrm{ddd}, J=12.2,11.1,3.9 \mathrm{~Hz}, 1 \mathrm{H}), 2.01$ (ddd, $J=13.3,3.9,1.9 \mathrm{~Hz}, 1 \mathrm{H}), 1.86$ (ddddd, $J=11.4,11.1,6.3,4.3,3.7 \mathrm{~Hz}, 1 \mathrm{H}), 1.53$ (ddd, $J=13.3$,

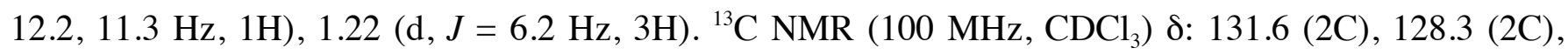
128.0, 123.3, 90.7, 82.2, 73.0, 69.9, 63.0, 43.3, 39.5, 30.2, 21.4. IR (KBr): 3399, 2936, $2918 \mathrm{~cm}^{-1}$. HRMS (DART) $m / z:[\mathrm{M}+\mathrm{H}]^{+}$calcd for $\mathrm{C}_{15} \mathrm{H}_{19} \mathrm{O}_{2}, 231.1385$; found, 231.1374. 9f': $R_{\mathrm{f}}=0.14$ (30\% EtOAc in $n$-hexane). ${ }^{1} \mathrm{H}$ NMR (500 MHz, $\left.\mathrm{C}_{6} \mathrm{D}_{6}\right)$ d: 7.42-7.36 (m, 2H), 7.03-6.96 (m, 3H), 3.95 (dqd, $J=11.1,6.2$, $1.9 \mathrm{~Hz}, 1 \mathrm{H}), 3.93(\mathrm{dd}, J=11.6,4.0 \mathrm{~Hz}, 1 \mathrm{H}), 3.67(\mathrm{dd}, J=11.6,11.0 \mathrm{~Hz}, 1 \mathrm{H}), 3.41(\mathrm{dd}, J=10.6,7.2 \mathrm{~Hz}$, $1 \mathrm{H}), 3.32(\mathrm{dd}, J=10.6,7.0 \mathrm{~Hz}, 1 \mathrm{H}), 2.97(\mathrm{ddd}, J=4.5,4.1,2.8 \mathrm{~Hz}, 1 \mathrm{H}), 1.79$ (ddddd, $J=11.0,7.2,7.0$, 4.1, 4.0 Hz, 1H), $1.67(\mathrm{ddd}, J=13.1,2.8,1.9 \mathrm{~Hz}, 1 \mathrm{H}), 1.32(\mathrm{ddd}, J=13.1,11.1,4.5 \mathrm{~Hz}, 1 \mathrm{H}), 1.14(\mathrm{~d}, J=$ $6.2 \mathrm{~Hz}, 3 \mathrm{H}$ ). HRMS (DART) $m / z:[\mathrm{M}+\mathrm{H}]^{+}$calcd for $\mathrm{C}_{15} \mathrm{H}_{19} \mathrm{O}_{2}, 231.1385$; found, 231.1386. 


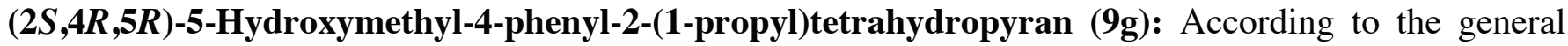
procedure C, compound $\mathbf{9 g}$ was obtained from $\mathbf{8 g}$ in $80 \%$ yield (2 steps). Colorless oil. Eluent for column: $16 \%$ EtOAc in $n$-hexane. $R_{\mathrm{f}}=0.24$ (30\% EtOAc in $n$-hexane). $[\alpha]_{\mathrm{D}}^{25}-14.4\left(c 1.07, \mathrm{CHCl}_{3}\right) .{ }^{1} \mathrm{H} \mathrm{NMR}$

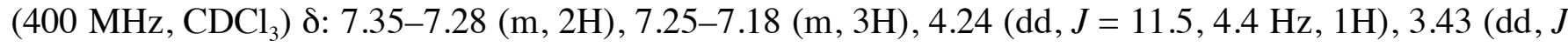
$=11.0,3.7 \mathrm{~Hz}, 1 \mathrm{H}), 3.43-3.36(\mathrm{~m}, 1 \mathrm{H}), 3.42(\mathrm{dd}, J=11.5,10.9 \mathrm{~Hz}, 1 \mathrm{H}), 3.26(\mathrm{dd}, J=11.0,7.0 \mathrm{~Hz}, 1 \mathrm{H})$, $2.60(\mathrm{ddd}, J=11.9,11.8,4.0 \mathrm{~Hz}, 1 \mathrm{H}), 2.00$ (ddddd, $J=11.9,10.9,7.0,4.4,3.7 \mathrm{~Hz}, 1 \mathrm{H}), 1.79(\mathrm{ddd}, J=$ $13.3,4.0,2.0 \mathrm{~Hz}, 1 \mathrm{H}), 1.51(\mathrm{ddd}, J=13.3,11.8,11.5 \mathrm{~Hz}, 1 \mathrm{H}), 1.60-1.32(\mathrm{~m}, 4 \mathrm{H}), 0.91(\mathrm{t}, J=7.0 \mathrm{~Hz}$, 3H). ${ }^{13} \mathrm{C}$ NMR (100 MHz, $\mathrm{CDCl}_{3}$ ) $\delta: 144.0,128.8$ (2C), 127.4 (2C), 126.7, 77.6, 70.9, 62.4, 44.3, 43.8, 40.1, 38.2, 18.6, 14.0. IR (KBr): 3449, 2957, $2930 \mathrm{~cm}^{-1}$. HRMS (DART) $m / z:[\mathrm{M}+\mathrm{H}]^{+}$calcd for $\mathrm{C}_{15} \mathrm{H}_{23} \mathrm{O}_{2}$, 235.1698; found, 235.1696.

\section{ACKNOWLEDGEMENTS}

This work was supported in part by JSPS KAKENHI Grant Numbers JP17H03998 and JP16K18847, by a grant from The Takeda Science Foundation, and by a grant for Basic Science Research Projects from The Sumitomo Foundation. We thank Arisa Sugiyama for technical assistance.

\section{REFERENCES AND NOTES}

1. (a) E. Quinoa, Y. Kakou, and P. Crews, J. Org. Chem., 1988, 53, 3642; (b) A. K. Ghosh and Y. Wang, J. Am. Chem. Soc., 2000, 122, 11027.

2. (a) G. R. Pettit, C. L. Herald, D. L. Doubek, D. L. Herald, E. Arnold, and J. Clardy, J. Am. Chem. Soc., 1982, 104, 6846; (b) M. Kageyama, T. Tamura, M. H. Nantz, J. C. Roberts, P. Somfai, D. C. Whritenour, and S. Masamune, J. Am. Chem. Soc., 1990, 112, 7407.

3. (a) D. Uemura, K. Takahashi, T. Yamamoto, C. Katayama, J. Tanaka, Y. Okumura, and Y. Hirata, J. Am. Chem. Soc., 1985, 107, 4796; (b) T. D. Aicher, K. R. Buszek, F. G. Fang, C. J. Forsyth, S. H. Jung, Y. Kishi, M. C. Matelich, P. M. Scola, D. M. Spero, and S. K. Yoon, J. Am. Chem. Soc., 1992, 114, 3162; (c) A. Ueda, A. Yamamoto, D. Kato, and Y. Kishi, J. Am. Chem. Soc., 2014, 136, 5171.

4. (a) P. A. Clarke and S. Santos, Eur. J. Org. Chem., 2006, 2045; (b) N. Kawai, J.-M. Lagrange, M. Ohmi, and J. Uenishi, J. Org. Chem., 2006, 71, 4530; (c) H. Yokoyama, G. Moriyama, K. Nishida, Y. Kusumoto, K. Tsuge, M. Miyazawa, and Y. Hirai, Heterocycles, 2018, 96, 470 and references cited therein.

5. (a) M. D. Lewis, J. K. Cha, and Y. Kishi, J. Am. Chem. Soc., 1982, 104, 4976; (b) J. A. Henderson, K. L. Jackson, and A. J. Phillips, Org. Lett., 2007, 9, 5299; (c) C.-G. Dong, J. A. Henderson, Y. Kaburagi, T. Sasaki, D.-S. Kim, J. T. Kim, D. Urabe, H. Guo, and Y. Kishi, J. Am. Chem. Soc., 2009, 131, 15642 . 
6. A. Ueda, T. Umeno, M. Doi, K. Akagawa, K. Kudo, and M. Tanaka, J. Org. Chem., 2016, 81, 6343.

7. After our report in 2016, O'Brien and co-workers reported a synthesis of 2,4,5-trisubstituted THPs by using similar effect of substituent at $\mathrm{C} 4$ position, although their synthesis is a racemic version and also substrates must involve a furan ring. D. A. Cooper, E. Robbins, G. J. Tizzard, S. J. Coles, and M. O’Brien, J. Org. Chem., 2017, 82, 3441.

8. (a) E. R. Jarvo and S. J. Miller, Tetrahedron, 2002, 58, 2481; (b) M. Nagano, M. Doi, M. Kurihara, H. Suemune, and M. Tanaka, Org. Lett., 2010, 12, 3564; (c) K. Akagawa and K. Kudo, Acc. Chem. Res., 2017, 50, 2429 and references cited therein.

9. The amount of benzoic acid was reduced for the ease of removal during purification and the concentration was changed to $0.4 \mathrm{M}$ to improve conversions. Under the above conditions, compound 3 was obtained from compound $\mathbf{2}$ in 97\% yield with 93\% ee.

10. Key NOE correlations were observed between H-2 and H-6 $6_{\mathrm{ax}}$, and between $\mathrm{H}-3_{\mathrm{ax}}$ and H-5.

11. (a) P. Deslongchamps, 'Stereoelectronic Effects in Organic Chemistry,' Pergamon Press, Inc., New York, 1983; (b) L. Ayala, C. G. Lucero, J. A. C. Romero, S. A. Tabacco, and K. A. Woerpel, J. Am. Chem. Soc., 2003, 125, 15521. 